Revue internationale P.M.E.

Économie et gestion de la petite et moyenne entreprise

Les relations interorganisationnelles des $P M E$, Katherine Gundolf et Annabelle Jaouen (dir.), Paris, Hermès-Lavoisier, 2008, 322 p.

\title{
Didier Chabaud
}

Volume 22, numéro 2, 2009

URI : https://id.erudit.org/iderudit/044035ar

DOI : https://doi.org/10.7202/044035ar

Aller au sommaire du numéro

Éditeur(s)

Presses de l'Université du Québec

ISSN

0776-5436 (imprimé)

1918-9699 (numérique)

Découvrir la revue

Citer ce compte rendu

Chabaud, D. (2009). Compte rendu de [Les relations interorganisationnelles des $P M E$, Katherine Gundolf et Annabelle Jaouen (dir.), Paris, Hermès-Lavoisier,

2008, 322 p.] Revue internationale P.M.E., 22(2), 173-177.

https://doi.org/10.7202/044035ar d'utilisation que vous pouvez consulter en ligne.

https://apropos.erudit.org/fr/usagers/politique-dutilisation/ 


\section{LES RELATIONS INTERORGANISATIONNELLES DES PME Katherine Gundolf et Annabelle Jaouen (dir.) Paris, Hermès-Lavoisier, 2008, 322 p.}

Quel est le rôle joué par les relations interpersonnelles (RI) dans les relations interorganisationnelles (RIO) des PME? C'est à l'étude de cette intéressante - et importante - question que nous convie l'ouvrage dirigé par Katherine Gundolf et Annabelle Jaouen (2008).

L'intérêt pour les relations interorganisationnelles et leur pilotage est récurrent en sciences de gestion, que l'on s'intéresse aux relations interorganisationnelles (Forgues, Fréchet et Josserand, 2006), aux réseaux (Josserand, 2007; Voisin, Ben Mahmoud-Jouini et Edouard, 2004) ou aux stratégies collectives (Yami, 2006; Yami et Le Roy, 2006). De même, l'interrogation sur le rôle des réseaux sociaux a connu d'amples développements, de la mise en évidence de «la force des liens faibles» (Granovetter, 1973) jusqu'à l'accent sur le capital social et son importance (Adler et Kwon, 2002; Burt, 2005). Mais, qu'en est-il lorsqu'on cherche à saisir les relations entre organisations ? Les réseaux sociaux - et les relations interpersonnelles - influencent-ils le déclenchement, le développement et le contenu - voire la performance - des relations interorganisationnelles, ou bien s'effacent-ils devant la logique de fonctionnement - le pouvoir et la règle, oserait-on risquer ${ }^{1}$ - des organisations?

La question est d'importance. Elle l'est d'autant plus dans un contexte (la PME) où la proximité joue un rôle central (Torrès, 2003, 2004) du fait de l'effet grossissant lié à la taille de l'organisation (Mahé de Boislandelle, 1996). L'analyse sera donc d'autant plus convaincante. S'agissant d'un ouvrage collectif, qui regroupe en 322 pages une douzaine de chapitres, outre une introduction et conclusion par les directrices, le moindre de leurs mérites n'est pas d'être parvenues à éclairer les diverses facettes de cette thématique. En effet, on va pouvoir à la fois tirer parti de la conception d'un véritable ouvrage collectif - avec un projet éditorial clair et suivi - et des articles qui y prennent place. Ceux-ci contribuent au projet collectif, à la façon d'un tableau impressionniste, l'intérêt résidant:

- dans chacune des contributions, avec de très beaux chapitres théoriques et/ou de belles études empiriques, la plupart des chapitres combinant à la fois mise en perspective théorique et étude qualitative stimulante, et

- dans le projet collectif et son résultat.

1. Pour reprendre Friedberg (1993). 
Conscients d'avoir «opté volontairement pour des études qualitatives, les auteurs ont choisi de privilégier l'analyse des processus, l'étude des relations en profondeur, la compréhension des situations et l'interprétation des discours» (Gundolf et Jaouen, p. 16). Ce choix permet de tirer de la lecture de chaque chapitre des connaissances nouvelles sur la thématique et/ou le cas.

L'exploration du thème s'effectue à travers des parties structurées autour des relations dyadiques (entre deux entreprises), autour des relations multi-acteurs (partie 2) et une dernière partie qui s'intéresse aux relations entretenues par le créateur d'entreprise avec son accompagnant ou son environnement lorsqu'il est un lifestyle entrepreneur. Le fait que les articles reviennent - à partir d'entrées théoriques et/ou de cas différents - sur des questions proches nous conduit à préférer une recension structurée autour de l'articulation entre RI et RIO à une vision linéaire.

Les relations interpersonnelles jouent un rôle important lorsqu'il s'agit de crédibiliser ou de légitimer les acteurs et ainsi d'instaurer la confiance. Tant l'origine que la dynamique ou le fonctionnement des relations interorganisationnelles sont alors impactés.

Tout d'abord, les relations interpersonnelles peuvent jouer un rôle de catalyseur: les individus qui partagent des RI désirent travailler ensemble. Sur ce point, bien souvent, sont présentées des success stories, dans lesquelles les RIO s'inscrivent avec succès dans la suite de RI. Gomez-Vélasco (chapitre 12) souligne ainsi que certains lifestyle entrepreneurs - ces entrepreneurs qui sont plus poussés par une quête de satisfaction personnelle que de gain économique - construisent leur projet sur la base de RI, tandis que Jaouen (chapitre 3) nous montre, dans une intéressante étude de 20 opérations d'alliances entre TPE, la diversité des rôles possibles des RI dans l'instauration de RIO. De même, plusieurs chapitres vont permettre d'affiner l'analyse et de saisir les dynamiques à l'œuvre à partir des RI, qu'il s'agisse du chapitre de Fourcade et al. - sur les systèmes agroalimentaires localisés (chapitre 8) - ou le chapitre 9 de Messeghem et Paradas sur le pôle européen d'innovation fruits et légumes (PEIFL), où le rôle des relations interpersonnelles semble quasiment premier dans l'émergence et le déploiement du projet collectif. Plus encore, la dimension interpersonnelle peut être centrale: ainsi Fonrouge et Sammut, après avoir clarifié le concept d'accompagnement dans le chapitre 11, montrent que l'interpersonnel - et la proximité entre l'accompagnant et l'entrepreneur -jouera un rôle crucial dans l'efficacité de l'accompagnement.

Si les RI peuvent être à l'origine de RIO, elles permettent également d'en améliorer le déroulement ou, plus largement, elles peuvent favoriser l'accès aux ressources. Ainsi que le soulignent Peyroux et Temri (chapitre 10), à l'aide de deux études de cas de TPE de haute technologie, les RI peuvent 
jouer sur la légitimité et l'accès aux ressources. Plus encore, les RI peuvent permettre d'équilibrer les RIO: ainsi, dans le chapitre de Meier et Missionnier (chapitre 2), le fait que les dirigeants des sociétés se connaissent et partagent des normes communes, va leur permettre de renouer des relations rompues sur une base d'égalité des sociétés - alors même que l'alliance est asymétrique, conclue entre entreprises de taille différente.

Cependant, les RI doivent être appréhendées de façon fine et dans la durée. Les RI peuvent ainsi se doubler d'une dimension affective: si la confiance préexiste, elle peut être mise à l'épreuve lorsque les relations interorganisationnelles conduisent à mettre au jour des intérêts divergents. Ici, le doute peut s'installer, les rancœurs, s'accumuler: en d'autres termes, l'affect peut prendre le dessus. L'étude de Gueguen et Pellegrin-Boucher (chapitre 4) est ici intéressante à méditer: alors que les partenaires se découvrent, que les liens se renforcent, la fusion des deux entreprises semble «rationnelle» (en termes d'efficacité économique). Cependant, lors de la mise en œuvre, l'attachement à l'entreprise familiale conduit l'un des partenaires à douter, puis à refuser la fusion. L'échec du rapprochement est patent et une tendance à la vendetta apparaît... On constate que lorsque les intérêts deviennent conflictuels ou lorsque les RI semblent aller à l'encontre des intérêts économiques, les modalités de résolution ne sont pas «évidentes» en fonction de la logique qui l'emporte. Dans certains cas, la solution se fera selon une logique économique, tandis que dans d'autres cas la solution sera marquée par l'affect, sacrifiant au besoin l'efficacité économique.

La vision est alors nuancée : les RI influencent les RIO, mais ne garantissent pas toujours le résultat. Le problème est renforcé lorsque la taille des réseaux ou des organisations augmente: lorsque l'on passe de relations dyadiques à des relations multi-acteurs, on pressent que la dimension interpersonnelle risque de devenir marginale. L'opportunisme individuel risque de réapparaître ou de se renforcer; les relations intuitu personae risquent de se déliter. Le chapitre 5 de de Freymann et Richomme-Huet souligne la façon dont les RI freinent de tels accords de coopération - incitant les acteurs à restreindre le champ de la coopération - ou en influencent la structuration, tandis que Gundolf (chapitre 7) montre à l'aide de l'étude de deux stations de sports d'hiver la diversité des rapports entretenus par les dirigeants: certains types d'entrepreneurs sont fortement impliqués dans le collectif, alors que d'autres adoptent des comportements instrumentaux (d'aucuns diraient «opportunistes ») à l'égard du collectif formé par la station de sports d'hiver. Enfin, le chapitre 7 de Amans et Loup montre comment la présence (ou l'absence) de mécanismes de contrôle social permet (ou non) d'assurer la viabilité d'un rapprochement à grande échelle dans le cas de réseaux constitués autour de deux centrales d'achats. 
L'interpersonnel supplante-t-il alors l'interorganisationnel? Une telle conclusion serait, au vu de la diversité des contributions, abusive. Les RI jouent un rôle important, mais elles n'expliquent en rien l'intégralité des observations. Ainsi que le soulignent Gundolf et Jaouen (p. 322), «si la logique tendrait à affirmer qu'elles [les RI] sont au cœur du bon fonctionnement des réseaux sociaux, voire des stratégies collectives, cela semble moins évident lorsque l'on s'intéresse aux alliances stratégiques (asymétriques ou non) ou aux rapprochements par acquisition notamment». En outre, «lorsque des jeux de pouvoir existent, que des liens de dépendance plus ou moins forts apparaissent entre des partenaires, que le projet commun est risqué et qu'une incertitude peut exister sur l'issue du partenariat, ou encore que les fruits de la coopération ne sont pas clairement définis ou répartis par anticipation, le risque d'opportunisme est fort» (id., p. 322). Une vision contingente ressort, qu'il convient de préciser.

On perçoit, ainsi, à la fois la richesse des analyses conduites - des résultats obtenus - ainsi que leur contingence. C'est un double mérite lié aux analyses qualitatives approfondies de souligner la complexité des situations et parfois la diversité des interprétations possibles, mais aussi à la qualité des éditrices que de n'avoir pas cherché à forcer le trait pour faire ressortir «une » vérité, ou à atténuer la divergence des résultats. S'il fallait formuler un regret, ce serait celui de l'absence d'un chapitre de synthèse, qui reprendrait précisément l'ensemble des apports, et des questions ouvertes par les divers chapitres du livre: un tel chapitre permettrait de faire écho au chapitre 1 de Michel Marchesnay, qui souligne combien la question du réseau a été pensée différemment selon les théories. Cela étant dit, le livre apparaît alors à la fois comme un outil essentiel pour faire le point sur la question des RI et des RIO, mais aussi comme un moyen efficace pour s'interroger sur les travaux à mener dans les années à venir. Somme toute, une lecture à la fois nécessaire et stimulante.

\author{
Didier Chabaud \\ Maître de conférences (HDR) \\ à l'Université Cergy-Pontoise \\ et professeur affilié à l'École \\ de management de Normandie
}

\title{
Bibliographie
}

ADLER, P.S. et S.Q. KWON (2002), «Social capital: prospects for a new concept», Academy of Management Review, vol. 27, n 1, p. 17-40.

BurT, R.S. (2005), Brokerage and Closure. An Introduction to Social Capital, Oxford, Oxford University Press. 
Forgues, B., M. FrÉCHET et E. JossERAND (2006), «Relations interorganisationnelles: conceptualisation, résultats et voies de recherche », Revue française de gestion, $\mathrm{n}^{\mathrm{o}} 164, \mathrm{p} .17-31$.

FRIEDBERG, E. (1993), Le pouvoir et la règle: dynamiques de l'action organisée, Paris, Seuil.

Granovetter, M. (1973), «The strength of weak ties», American Journal of Sociology, vol. 78, p. 1360-1380

JOSSERAND, E. (2007), «Le pilotage des réseaux. Fondements des capacités dynamiques de l'entreprise », Revue française de gestion, n 170, p. 95-102.

MAHÉ DE Boislandelle, H. (1996), «Effet de grossissement et management des ressources humaines en $\mathrm{PME}$ », $\mathrm{III}^{\mathrm{e}}$ Congrès international francophone $\mathrm{PME}$ (CIFPME), Trois-Rivières, Québec.

TORRÈs, O. (2003), «Petitesse des entreprises et grossissement des effets de proximité», Revue française de gestion, vol.144, p. 119-138.

TORRÈs, O. (2004), Essai de théorisation de la gestion des PME: de la mondialisation à la proxémie, Mémoire d'habilitation à diriger des recherches en sciences de gestion, IAE de Caen, <http://www.oliviertorres.net/travaux/pdf/hdrtorres.pdf>.

Voisin, C., S. Ben Mahmoud-Jouini et S. Edouard (dir.) (2004), Les réseaux: dimensions stratégiques et organisationnelles, Paris, Economica.

YAMI, S. (2006), «Les stratégies collectives à l'épreuve des faits », Revue française de gestion, vol. 167, p. 87-90.

YAMI, S. et F. LE ROY (2006), Les stratégies collectives: rivaliser et coopérer avec ses concurrents, Colombelles, Éditions EMS, 409 p. 